\title{
Adult Patient Presenting Rare Brain Tumour-Gliosarcoma
}

\author{
Khalequezzaman $\mathrm{S}^{1}$, Alam J², Ahsan $\mathrm{S}^{3}$, Khan $\mathrm{BS}^{4}$
}

\begin{abstract}
Gliosarcoma is a rare primary malignancy of the central nervous system, classified by the World Health Organization as a high-grade glioma and a variant of glioblastoma multiforme. A 57-year-old gentleman presented with a history of left-sided weakness and loss of appetite. Brain MRI was suggestive of right frontal and thalamic mass lesion with contrast enhancement at the periphery. Open biopsy examination revealed a malignant brain tumour presenting a biphasic tissue pattern with gliomatous and mesenchymal components suggestive of gliosarcoma. Although the treatment of gliosarcomas is almost similar to glioblastomas (surgical resection and, depending on clinical status, radiotherapy and/or chemotherapy) the prognosis of gliosarcomas remains poor.
\end{abstract}

Key words

Brain tumor, gliosarcoma

\section{Introduction}

Gliosarcoma is a rare primary malignancy of the central nervous system, classified by the World Health Organization (WHO) as a high-grade glioma and a variant of glioblastoma multiforme. ${ }^{1}$ Gliosarcoma accounts for $<2 \%$ of all gliomas and $5 \%$ of all astrocytomas. The tumour has a clinical presentation, natural history, and radiological profile similar to glioblastoma multiforme.

\section{Case Report}

A 57 years old right handed gentleman admitted in Apollo Hospitals Dhaka with the complains of difficulty in moving left lower limb for 1 month and loss of appetite for 2 months duration. He gave the history of fall 2 months back. He was hypertensive and non diabetic. No history of loss of consciousness or convulsion. At admission, his GCS scores were E4V5M6, muscle power was $3 / 5$ on left side, $5 / 5$ on right side.

Investigations at admission were normal for blood CBC, S. Creatinine, S. electrolytes, liver function tests and lipid profile.

He underwent MRI of brain which revealed 3.3 x 3.1 x $3.9 \mathrm{~cm}$ well defined lesion in right thalamic region with mild perifocal edema. The mass is hypointense on T1 WI and hyperintense

1. Specialist, Dept. of Radiology \& Imaging, Apollo Hospitals Dhaka 2. Senior Consultant, Dept. of Radiology \& Imaging, Apollo Hospitals Dhaka 3. Consultant, Dept. of Radiology \& Imaging, Apollo Hospitals Dhaka 4. Consultant, Dept. of Radiology \& Imaging, Apollo Hospitals Dhaka 
on T2 WI with hypointense rim (Fig.1-a,b). After contrast, which showed irregular rim enhancement and an enhancing thin septation (Fig.1-d). The mass causes mass effect as evidenced by compression on body of right lateral and $3^{\text {rd }}$ ventricles with mild obstructive triventriculomegaly. Another smaller similar lesion was noted in right frontal lobe. In

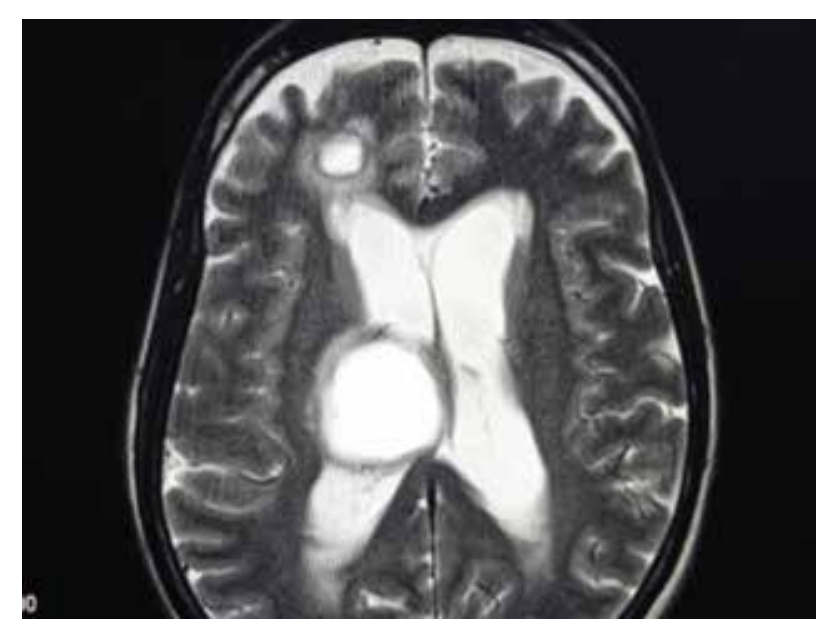

1a

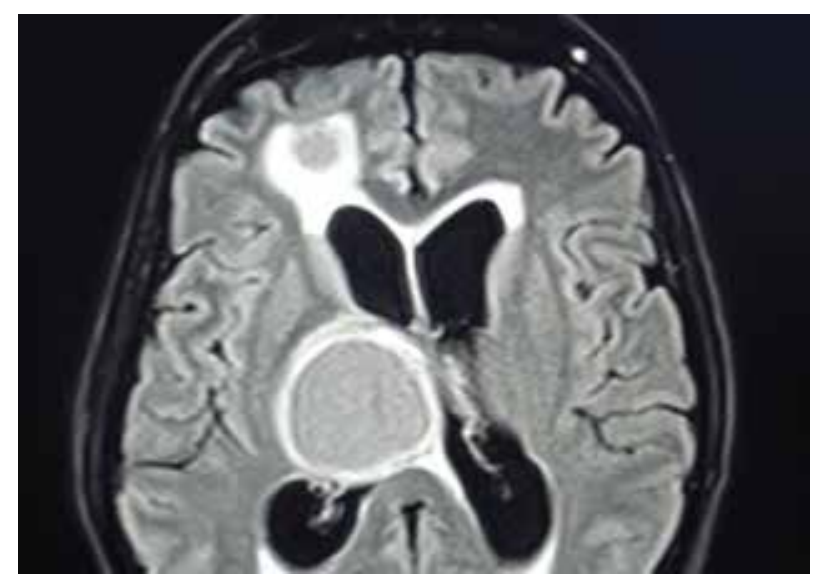

1c
Diffusion weighted (DW) and Apparent Diffusion Co-efficient (ADC) sequences, cystic area was similar to CSF signal intensity (Fig. 2-a,b).

The conclusion of MRI examination was drawn as multi focal gliomas, however, other possibility of metastases could not be ruled out.

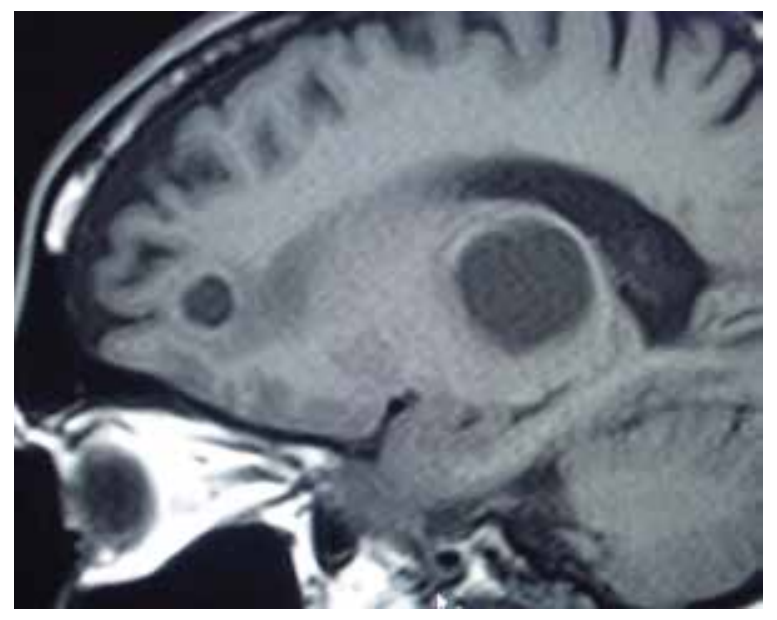

1b

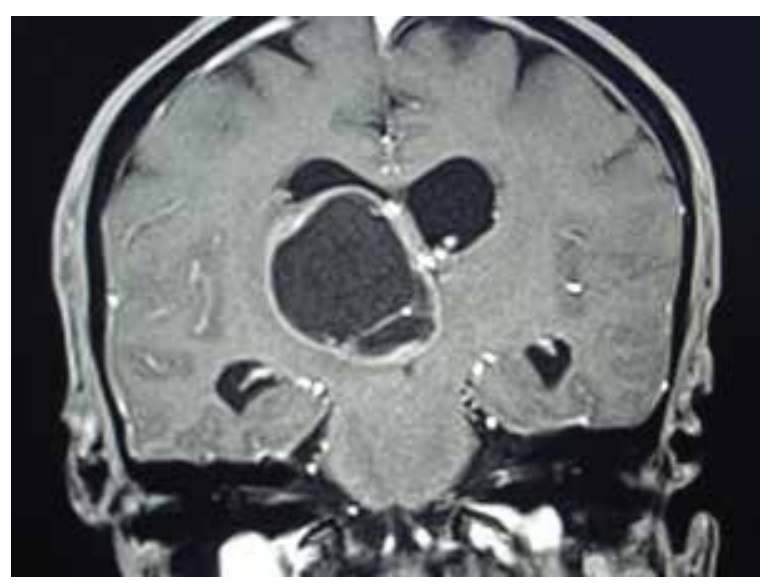

1d

Fig.1: (a) T1 WI (b) T2 WI and (c) FLAIR sequences showing two similar rounded lesions in right thalamic region and frontal region. The mass is hypointense on T1WI and hyperintense on T2WI and FLAIR sequence. After contrast (d), it shows rim enhancement and enhancing thin septa within 


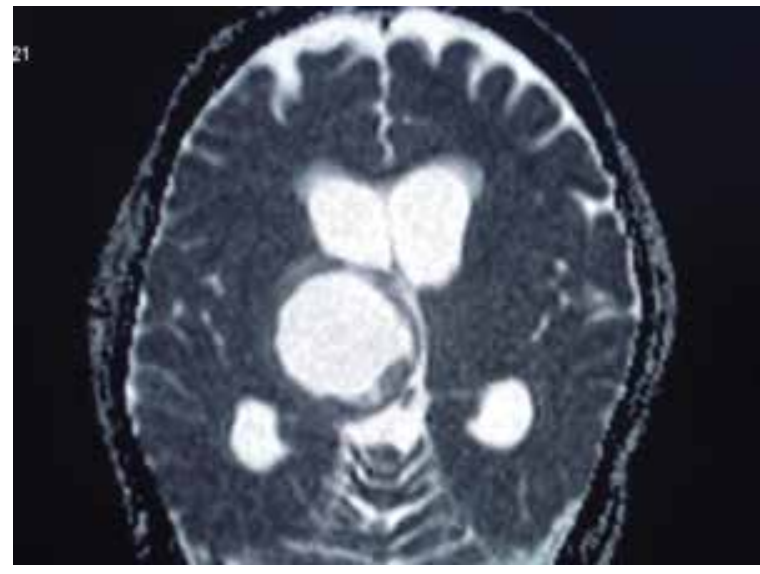

2a

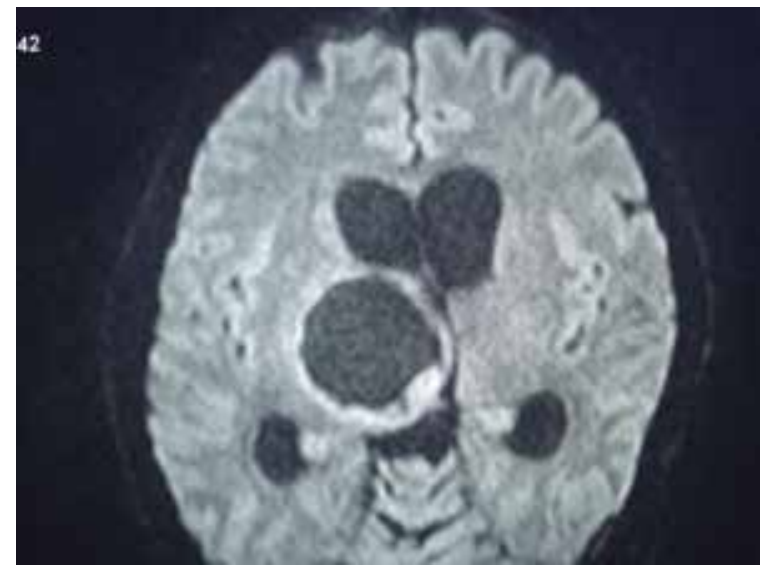

2b

Fig. 2: (a) DW and (b) ADC sequences showing cystic area having similar signal intensity as CSF

Patient underwent CT scan of abdomen to search for any primary cause or metastases, but revealed no abnormality. His chest X-ray was unremarkable. PSA was within normal limit (1.3 $\mathrm{ng} / \mathrm{ml})$.

For uncertain diagnostic dilemma, patient underwent right fronto-parietal craniotomy and open biopsy of the right thalamic lesion was taken under GA. The material was grey brown. At biopsy, the section showed proliferation of anaplastic tumor cells displaying gliomatous as well as sarcomatous differentiation along with transition area. The spindle cells were forming fascicles and at places adenoid arrangement. The cells showed hyperchromatic nuclei with frequent mitoses and focal areas of necrosis. Gliosarcoma, WHO grade - IV, was diagnosed. The patient was discharged in a hemodynamically stable condition and advised to consult with radiotherapist as patient needs radiotherapy.

\section{Discussion}

According to the new WHO classification, gliosarcoma is defined as a glioblastoma variant, characterized by a biphasic tissue pattern with alternating areas displaying glial and mesenchymal differentiation. ${ }^{1}$ Gliosarcoma is a relatively rare malignant neoplasm accounting for approximately $2 \%$ of glioblastomas. ${ }^{2}$ Gliosarcoma occurs most commonly in adults in the fourth to sixth decades of life and men are more commonly affected than women (ratio 1.8:1). Anatomically, gliosarcomas are usually located in the cerebral cortex, and involve the temporal, frontal, parietal, and occipital lobes in decreasing frequency. ${ }^{3}$ The presenting symptoms depend on the location of the tumour. The aetiology of gliosarcoma remains uncertain, although it is recognized that gliomas can induce sarcomatous transformation in the supporting mesenchymal elements and irradiation of the central nervous system can induce malignant transformation of the brain parenchyma and the meninges, predominantly to fibrosarcoma. ${ }^{4}$ The appearance of gross gliosarcoma may be a poorly delineated 


\section{CASE REPORT}

peripheral greyish tumour mass with central yellowish necrosis stippled with red and brown from hemorrhage, and with the sarcomatous component producing a firm discrete mass. Histologically, the diagnosis of gliosarcoma is based on a biphasic tissue pattern comprising 2 distinct malignant cell populations, one component being gliomatous (fulfilling the criteria for glioblastoma) and the other with malignant mesenchymal differentiation (fulfilling the criteria for sarcoma). The glioblastoma part of the tumour forms heterogeneous infiltrative areas with hemorrhage and necrosis. The sarcomatous portion produces a firm discrete mass. At microscopy, the glial portions show the typical features of glioblastoma multiforme. Interestingly, the sarcomatous component can have varied histological features, ranging from the herringbone pattern of fibro-sarcoma to the malignant bone of an osteosarcoma and cartilaginous differentiate of chondrosarcoma. ${ }^{5}$ Single institutional data from Germany published in 2009 revealed only 16 patients treated over a period of 10 years (1997-2006). ${ }^{6}$ Another audit from an institute of India published in 2008 presents only 24 patients of gliosarcoma as compared to 251 cases of GBM treated over a period of 15 years (1990-2004). ${ }^{7}$ 16 cases of gliosarcoma was reported from pathology department of Aga Khan University hospital, Karachi, Pakistan in 2004. ${ }^{8}$ There are fewer than 20 reported cases of extracranial metastases of gliosarcoma with majority of them reflecting a tendency for haematogenous dissemination.

\section{Reference}

1. Ohgaki H, Biernat W, Reis R, Hegi M, Kleihues P. Gliosarcoma.

In: Kleihues P, Cavenee WK, editors. Pathology and genetics of tumors of the nervous system. 2nd ed.

Lyon: IARC Press; 2000. p 42-4.

2. Lieberman KA, Fuller CE, Caruso RD, Schelper RL. Postradiation gliosarcoma with osteosarcomatous component. Neuroradiology. 2001;43:555-8.

3. Galansis E, Buckner JC, Dinapoli RP, Scheithauer BW, Jenkin RB, Wang CH. Clinical outcome of gliosarcoma compared with glioblastoma multiforme: North Central Cancer Treatment Group results. J Neurosurg. 1998;89:425-30.

4. Feigin I, Allen LB, Lipkin L, Gross SW. The endothelial hyper-plasia of cerebral blood vessels with brain tumor and its sarcomatous transformation. Cancer. 2006;11:264-77.

5. Wrinkler PA, Buttner A, Tomezzoli A, Weis S. Histologically repeatedly confirmed gliosarcoma with long survival: review of the literature and report of a case. Acta Neurochir. 2000;142:91-5.

6. Buhl R, Stark AM, Hugo HH, Rohr A, Mehdorn HM. Gliosarcoma: clinical experiences and additional information with MR spectroscopy. Neurol Res. 2009;31:873-7.

7. Kumar P, Singh S, Kumar P, Krishnani N, Datta NR. Gliosarcoma: an audit from a single institution in India of 24 post-irradiated cases over 15 years. J Cancer Res Ther. 2008;4:164-8.

8. Ahmed Z, Azad NS, Muzaffer S, Nasir I, Hasan S. CNS tumors at AKU: an update plus a brief discussion on intraventricular tumors with special emphasis on central neurocytoma. J Ayub Med Coll Abbottabad. 2004;16:12-5. 